\title{
ASYMPTOTIC FORMULAS FOR SOME ARITHMETIC FUNCTIONS
}

\author{
P. Erdös
}

(received April 9, 1958)

Let $f(x)$ be an increasing function. Recently ${ }^{1)}$ there have been several papers which proved that under fairly general conditions on $f(x)$ the density of integers $n$ for which $(n, f(n))=1$ is $6 / \pi^{2}$ and that $(d(n)$ denotes the number of divisors of $n$ )

$$
\sum_{n=1}^{x} d(n,[f(n)])=\left((1+o(1)) \pi^{2} x / 6\right.
$$

In particular both of these results hold if $f(x)=x^{\alpha}, 0<\alpha<1$ and the first holds if $f(x)=[\alpha x], \alpha$ irrational.

In this note we are going to prove the following:

THEOREM 1. The necessary and sufficient condition that for an irrational $\alpha$ we should have

(1) $\sum_{n=1}^{x} d(n,[\alpha n])=(1+o(1)) \pi^{2} x / 6$

is that for every $c>0$ the number of solutions of

$$
\alpha<a / b<\alpha+1 /(1+c)^{b}
$$

should be finite in positive integers $a$ and $b$.

$$
\text { Denote } \sigma(n)=\sum_{\mathrm{d} \mid \mathrm{n}} \mathrm{d} \text {. It is easy to see that for } 0<\alpha<\frac{1}{2}
$$

(3) $\sum_{n=1}^{x} \sigma\left(n,\left[n^{\alpha}\right]\right)=(1+o(1)) \times \log x$

Very likely (3) also holds for $1 / 2<\alpha<1$ but I have not yet been able to show this. By more complicated arguments I can show

Can. Math. Bull., vol. 1, no. 3, Sept. 1958 
THEOREM 2. The necessary and sufficient condition that for an irrational $\alpha$ we should have

(4) $\sum_{n=1}^{x} \sigma(n,[n \alpha])=(1 / 2+o(1)) x \log x$

is that for every $\varepsilon>0$ the number of solutions in positive integers $a$ and $b$ of

$$
|\alpha-a / b|<\frac{1}{b^{2+\varepsilon}}
$$

and of

(6) $\alpha<a / b<\alpha+\varepsilon b^{-2} / \log b$

should be finite.

It is easy to see that conditions (5) and (6) are equivalent to the following: Put $\alpha=a_{0}+\frac{1}{a_{1}+} \frac{1}{a_{2}+} \cdots$, then

$$
(1 / n) \log a_{n} \rightarrow 0,(1 / n) a_{2 n+1} \rightarrow 0
$$

In the present note we will not prove Theorem 2 since the proof is similar to that of Theorem 1 , but is rather more complicated. for

Similarly one could try to obtain an asymptotic formula

$$
\sum_{n=1}^{x} \sigma(n,[f(n)])
$$

for more general functions $f(x)$, but I have not succeeded in obtaining any interesting results.

Now we prove Theorem 1 . Denote by $N(y, 1 / k)$ the number of integers $1<\mathrm{n}<\mathrm{y}$ for which

$$
\begin{aligned}
& 0<n \alpha-\left[\begin{array}{ll}
n & \alpha
\end{array}\right]<1 / k \\
& \left(n,\left[\begin{array}{ll}
n & \alpha
\end{array}\right]\right) \equiv 0(\bmod k) \text { holds if and only if } n=v k \text { and } \\
& \quad v k \alpha=u k+\theta, 0<\theta<1,
\end{aligned}
$$

that is $(n,[n \alpha]) \equiv 0(\bmod k)$ holds if and only if

$$
0<\mathrm{v} \alpha-[\mathrm{v} \alpha]<1 / \mathrm{k} \text {. }
$$


Thus the number of integers $n<x$ satisfying $(n,[n \alpha]) \equiv 0$ $(\bmod k)$ equals $N(x / k, 1 / k)$, ( since $n-v k$ implies $v<x / k)$. Thus by interchanging the order of summation

(7) $\sum_{n=1}^{x} d\left(n,\left[\begin{array}{ll}n & \alpha\end{array}\right]\right)=\sum_{k=1}^{x} N(x / k, 1 / k)$.

Since $n \alpha-[n \alpha]$ is equidistributed $(\bmod 1)$ we evidently have (8) $\mathrm{N}(\mathrm{x} / \mathrm{k}, 1 / \mathrm{k})=(1+\mathrm{o}(1))\left(\mathrm{x} / \mathrm{k}^{2}\right)$,

for fixed $k$ as $x$ tends to infinity. Thus from (7) and (8) for every irrational $\alpha$

(9) $\sum_{n=1}^{x} d\left(n,\left[\begin{array}{ll}n & \alpha\end{array}\right]\right) \geq(1+o(1)) \sum_{k=1}^{\infty} x / k^{2}=(1+o(1)) h^{2} x / 6$

Assume now that (2) is not satisfied. Then there is a fixed $c>0$ and arbitrarily large values of $b$ for which

(10) $\alpha<a / b<\alpha+1 /(1+c)^{b}$.

Put $(1+c)^{b}=x$. Write

(1I) $\sum_{n=1}^{x} d\left(n,\left[\begin{array}{ll}n & \alpha\end{array}\right]\right)=\sum_{1}+\Sigma_{2}$

where in $\Sigma_{1}, n \neq 0(\bmod b)$ and in $\Sigma_{2}, n \equiv 0(\bmod b)$. From the equidistribution of $n \alpha .-[n \alpha]$ it follows that for fixed $k$ the number of integers satisfying

$$
1<\mathrm{n}<\mathrm{x}, \quad \mathrm{n} \neq 0(\bmod \mathrm{b}), 0<\mathrm{n} \alpha-[\mathrm{n} \alpha]<1 / \mathrm{k}
$$

is not less than

(12) $N(x / k, 1 / k)-x / b=(1+o(1)) x / k^{2}-x / b$.

Thus from (7) and (12) we have for every fixed $t$

(13) $\sum_{1}>\sum_{k=1}^{t}\left((1+o(1)) x / k^{2}\right)-t x / b=(1+o(1)) \pi^{2} x / 6$ we have In $\Sigma_{2}, \mathrm{n}=\mathrm{vb} \leq \mathrm{x}$. Thus from $(10)$ and $\mathrm{vb} \leq \mathrm{x},(1+\mathrm{c})^{\mathrm{b}}=\mathrm{x}$

$$
[\mathrm{n} \alpha]=[\mathrm{vb} \alpha]=\left[\mathrm{va}+\theta \mathrm{vb} /(1+\mathrm{c})^{\mathrm{b}}\right]=\mathrm{va}(0<\theta<1)
$$

Thus $(v b,[v b \alpha]) \equiv 0(\bmod v)$ for all $1 \leq v<x / b$. Hence 


$$
\text { (14) } \begin{aligned}
\Sigma_{2} \geqslant \sum_{1 \leq v<\mathrm{x} / \mathrm{b}} \mathrm{d}(\mathrm{v}) & =(1+o(1))(\mathrm{x} / \mathrm{b}) \log (\mathrm{x} / \mathrm{b}) \\
& =(1+o(1)) \mathrm{x} \log (1+\mathrm{c})
\end{aligned}
$$

Now (11), (13) and (14) show that (1) does not hold. Thus (2) is a necessary condition for the validity of (1).

To show that $(2)$ is sufficient we need an upper estimation for $N(x / k, 1 / k)$ for large $k$. Put $x / k=y:$ it is well known that there exists an $a / b$ satisfying

$$
|\alpha-a / b|<1 /(2 b y), b<2 y,(a, b)=1 .
$$

Now we distinguish two cases. First as sume $b \geq k / 2$. Clearly for $\mathrm{l} \leq \mathrm{n} \leq \mathrm{y}$

$$
n \alpha-[n \alpha]=u / b+\theta / b, \quad|\theta|<1 / 2 .
$$

Thus $0<\mathrm{n} \alpha-[\mathrm{n} \alpha]<1 / \mathrm{k}$ can only hold if $\mathrm{u}=0,1, \ldots, \mathrm{z}+1$ where

$$
z / b \leq 1 / k<(z+1) / k \text {, or } z \leq b / k .
$$

The number of $n^{\prime} s$ not exceeding $y$ for which $u$ has a given value is clearly less than $2 y / b+1$. Thus from (17) and $b \geq k / 2$ we have

(18) $N(x / k, 1 / k)<(b / k+1)(2 y / b+1) \leq(3 b / k)(4 y / b)=12 x / k^{2}$.

Next assume $b<k / 2$. If $a / b<\alpha$ then $N(x / k, 1 / k)=0$ since in (16) $\theta \leq 0$, thus for $u=0 n \alpha-[n \alpha]$ is not in $(0,1 / k)$ and for $u=1 \bar{n} \alpha-[\mathrm{n} \alpha]>1 / 2 b>1 / k$.

Thus $a / b>\alpha$. Clearly $0<n \alpha-[n \alpha]<1 / k$ is only possible if $u=0$, that is if $n \equiv 0(\bmod b)$. Thus

(19) $N(x / k, 1 / k) \leqslant(x /(b k)$.

If $\mathrm{N}(\mathrm{x} / \mathrm{k}, 1 / \mathrm{k})>0$, then ( ince all the $\mathrm{n}<\mathrm{x} / \mathrm{k}$ for which $0<n \alpha-[n \alpha]<1 / k$ are multiples of b) we have by (15)

$$
b \alpha-[b \alpha]<\min (k / x, 1 / k) \leq x^{-1 / 2},
$$

but this implies by (2) that

(20) b/log $x \rightarrow \infty$. 
Thus finally from (7), (8), (18) and (19) we have for every fixed $t$

$$
\sum_{n=1}^{x} d(n, \quad n \alpha) \leq(1+o(1)) \pi^{2} x / 6+12 \times \sum_{k>t}\left(1 / k^{2}\right)+(x / b) \sum_{k<x} \frac{1}{k}
$$

(21)

$$
\sum_{n=1}^{x} d(n,[n \alpha]) \leqslant(1+o(1)) \pi^{2} x / 6 .
$$

From (9) and (21) we have that if (2) is satisfied, then

$$
\sum_{n=1}^{x} d(n,[n \alpha])=(1+o(1)) \pi^{2} x / 6 .
$$

Thus condition (2) is sufficient, which completes the proof of our Theorem.

\section{University of British Columbia}

1) See G.L. Watson, Canadian Journal of Math. 5(1953), 451-455, T. Estermann, ibid 5(1953), 456-459 and J. Lambek and L. Moser, ibid 7(1955), 155-158. See also a forthcoming paper by P. Erdós and G.G. Lorentz in Acta Arithmetica.

\section{CORRECTION}

In the paper "On an elementary problem in number theory" by Paul Erdös in Vol.1, no. 1 of this Bulletin, P. 5 , line 5 should read

$$
0 \leqslant u, v<f(x) \text { and }(x+u, y+v) \neq 1
$$

